\title{
Defect Characterization in Irradiated Nanocrystalline Materials via Automated Crystal Orientation Mapping
}

Pranav K. Suri, ${ }^{1}$ James E. Nathaniel, ${ }^{1}$ Christopher M. Barr, ${ }^{1,2}$ Jon K. Baldwin ${ }^{3}$, Khalid Hattar, ${ }^{2}$ and Mitra L. Taheri ${ }^{1}$

1. Department of Materials Science and Engineering, Drexel University, Philadelphia, PA 19104

2. Ion Beam Laboratory, Sandia National Laboratories, Albuquerque, NM 87185

3. Center for Integrated Nanotechnologies, Los Alamos National Laboratory, Los Alamos, NM 87545

Nanocrystalline metals and alloys are potential candidates for next generation nuclear reactors due to a high volume fraction of grain boundaries, which can act as efficient sinks for irradiation induced defects [1,2]. Present research is focused on understanding the annihilation and evolution of defects adjacent to grain boundaries and interfaces in irradiated materials $[3,4]$. Traditionally, transmission electron microscopy (TEM) has played a critical role in the evaluation of irradiation induced vacancy and interstitial dislocation loop size and density [5]. There are conventional methods of imaging defects in crystalline materials via TEM such as two-beam dynamical condition, down-zone imaging, and weak-beam dark-field microscopy which requires tilting of the specimen along desired crystal orientations [5]. However, specimen tilting along a definite crystal orientation is not easily achievable in nanocrystalline metals and alloys with grain sizes smaller than $100 \mathrm{~nm}$. With the advent of automated crystal orientation mapping (ACOM) combined with the routine use of stable field-emission gun electron microscopes, it is now possible to map crystal orientations down to a nanometer spatial resolution without specimen tilting [6]. Here, we describe our efforts to develop new methods of defect characterization in nanocrystalline materials combining the use of ACOM with the conventional TEM defect imaging methods without employing specimen tilting. We explore the various defect imaging techniques and access the suitability of each technique for imaging defects in nanocrystalline materials. We use JEOL 2100F TEM operated at $200 \mathrm{kV}$ having NanoMegas ASTAR precession diffraction system installed on it for all our experiments. We employ irradiated coarse and nanocrystalline grain $\mathrm{Ni}-5 \mathrm{wt} \% \mathrm{Cr}$ as our face-centered cubic model alloy system. Coarse and nanocrystalline grain specimens were irradiated at $20 \mathrm{MeV}$ and $1.2 \mathrm{MeV}$, respectively, with $\mathrm{Ni}^{4+}$ ions in Ion Beam Laboratory at Sandia National Laboratories.

The combined defect imaging and ACOM experiments on both coarse and nanocrystalline grain specimens show that there are specific grain orientations, as color coded by the inverse pole figure coloring scheme, which provide an easy route of finding grains ideally suited for defect characterization in the nanocrystalline specimen without any tilting. For example, Figure 1 shows the presence of irradiation induced defects in coarse grain $\mathrm{Ni}-5 \mathrm{wt} \% \mathrm{Cr}$ via down-zone bright-field (BF) scanning TEM (STEM) along [011] zone-axis (ZA) and two-beam dynamical condition for imaging with $\boldsymbol{g}=200$ from [011] ZA. The color code from ACOM for the two-beam dynamical condition is depicted in the inset of Figure 1(right panel). This specific color code grain when observed in the nanocrystalline specimen, provide a favorable defect imaging condition (Figure 2). The implications for these on directly imaging defects in nanocrystalline metals and alloys via TEM and ACOM, and comparison of these results with defect imaging on other crystal orientations of $\mathrm{Ni}-5 \mathrm{wt} \% \mathrm{Cr}$ will be discussed [7]. 
References:

[1] G. Ackland, Science, 327 (2010), 1587.

[2] I. J. Beyerlein et al., Mater. Today 16 (2013), 443.

[3] X. -M. Bai et al., Science 327 (2010), 1631.

[4] I. J. Beyerlein et al., Prog. Mater. Sci. 74 (2015), 125.

[5] M. L. Jenkins and M. A. Kirk, Characterization of Radiation Damage by Transmission Electron Microscopy, (CRC Press, 2000).

[6] E. F. Rauch and M. Véron, Mater. Charact. 98 (2014), 1.

[7] This work at the Drexel University is supported by the United States Department of Energy, Basic Energy Sciences under the Early Career program through contract DE-SC0008274. This work was performed, in part, at the Center for Integrated Nanotechnologies, an Office of Science User Facility operated for the U.S. Department of Energy (DOE) Office of Science. Sandia National Laboratories is a multi-program laboratory managed and operated by Sandia Corporation, a wholly owned subsidiary of Lockheed Martin Corporation, for the U.S. Department of Energy's National Nuclear Security Administration under contract DE-AC04-94AL85000.
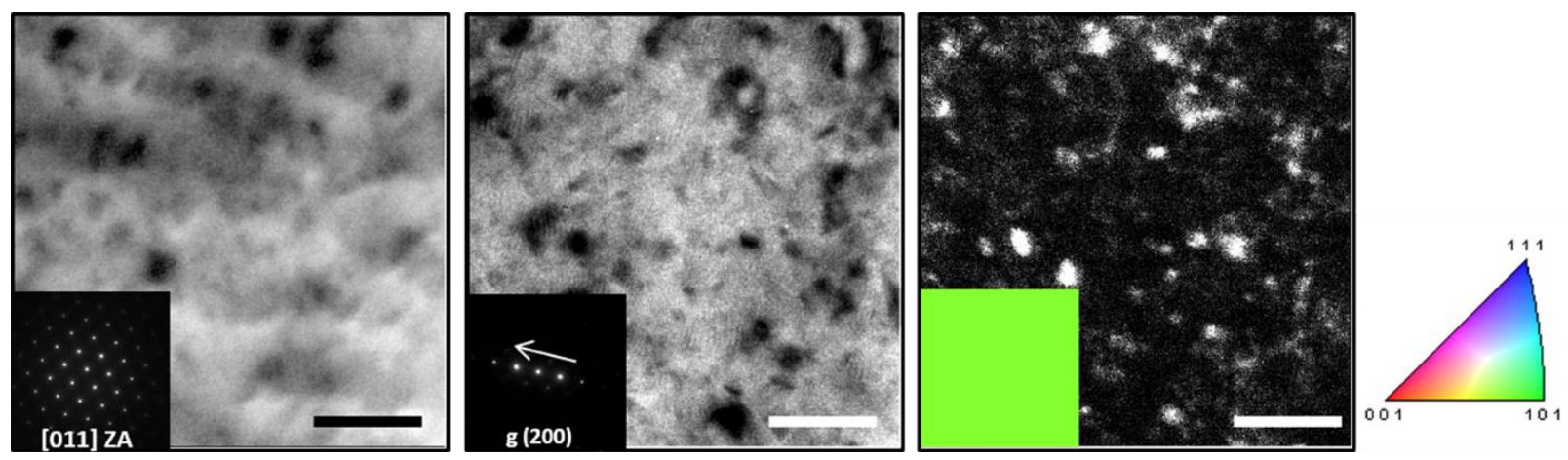

Figure 1. (Left Panel) BF-STEM image and SADP (inset) of irradiated coarse grain Ni-5wt\% $\mathrm{Cr}$ along the [011] ZA. (Center Panel) BF image, acquired under two-beam dynamical condition from [011] ZA with $g=200$, from the same area as in BF-STEM image and the inset shows the twobeam condition for this image acquisition. (Right Panel) DF image, acquired under two-beam dynamical condition from [011] ZA with $g=200$, from the same area as in BF-STEM image and the inset shows ACOM of the acquisition area. Scale bar is $25 \mathrm{~nm}$ in all the images.
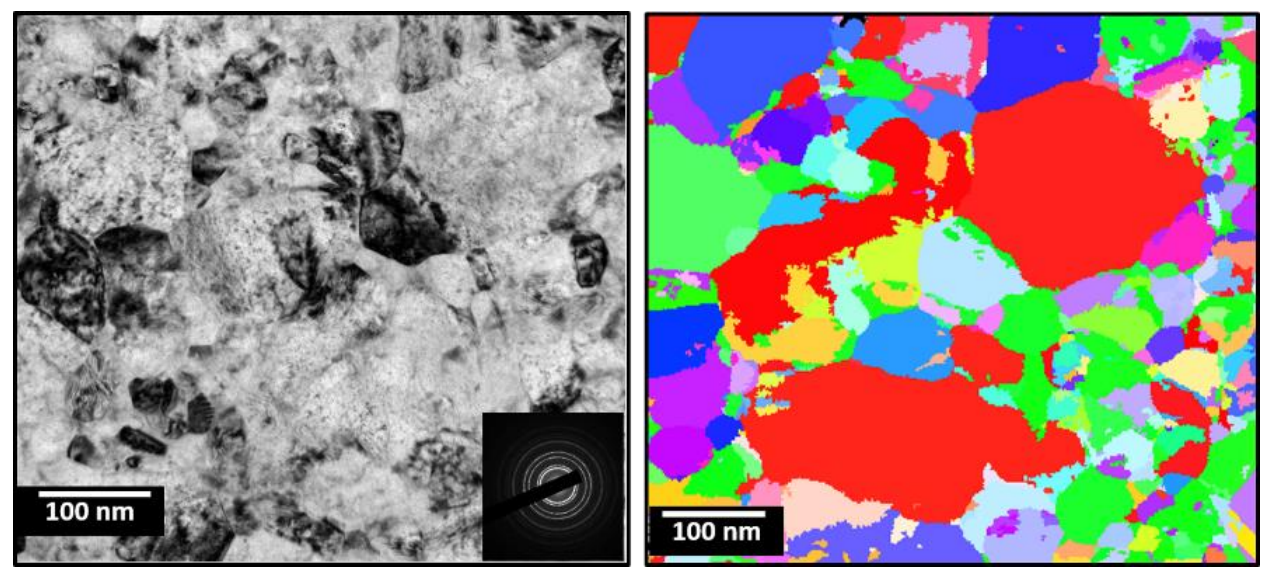

Figure 2. (Left Panel) BF-TEM image and SADP (inset) of irradiated nanocrystalline Ni-5wt\%Cr. (Right Panel) ACOM from the same region as shown in the left panel. 